\title{
A Case of Labyrinthine Fistula by Cholesteatoma Mimicking Lateral Canal Benign Paroxysmal Positional Vertigo
}

\author{
Dae Bo Shim, Kyung Min Ko, Mee Hyun Song, and Chang Eun Song \\ Department of Otorhinolaryngology, Myongji Hospital, Goyang, Korea
}

\author{
Received October 31, 2014 \\ Revised November 27, 2014 \\ Accepted November 28, 2014
}

\author{
Address for correspondence \\ Chang Eun Song, MD \\ Department of Otorhinolaryngology, \\ Myongji Hospital, \\ 55 Hwasu-ro 14beon-gil, \\ Deogyang-gu, Goyang 412-270, \\ Korea \\ Tel +82-31-810-5451 \\ Fax +82-31-969-0500 \\ E-mail entsong@gmail.com
}

Acute peripheral vestibulopathy, of which the chief complaint is positional vertigo, comprises benign paroxysmal positional vertigo (BPPV), labyrinthitis, labyrinthine fistula, and cerebellopontine angle tumors. Since the typical presentation of labyrinthine fistulas may be sensorineural hearing loss, positional vertigo, or disequilibrium, it is often difficult to distinguish from BPPV or Meniere's disease. Herein we report a 61-year-old female patient with typical symptoms and signs attributable to geotropic type variant of the lateral semicircular canal BPPV on the left side, who eventually was confirmed as having a labyrinthine fistula from chronic otitis media with cholesteatoma on the left side. This is another case where, even in the presence of isolated vertigo showing typical findings of acute peripheral vestibulopathy, other otologic symptoms and signs must not be overlooked.

Korean J Audiol 2014;18(3):153-157

\section{Introduction}

The most common peripheral vestibulopathies diagnosed from positional vertigo may include benign paroxysmal positional vertigo (BPPV), labyrinthitis, and acute vestibular neuritis, and less commonly labyrinthine fistula or cerebellopontine angle tumors. ${ }^{1)}$ Labyrinthine fistula, in particular, is reported to rise from 3.6 to $13.9 \%$ of chronic otitis media with cholesteatoma, and known to affect the lateral semicircular canal most frequently. ${ }^{2,3)}$ Typical symptoms of labyrinthine fistula are positional vertigo, severe disequilibrium, and sensorineural hearing loss, collectively making it difficult to differentiate from BPPV or Meniere's disease. ${ }^{4)}$ Confounding the distinction is the sensitivity of the fistula test, of which is reported to be between 30 to $60 \%$ at best. High resolution computed tomography of the temporal bone (TBCT) may help diagnose fistulas with sensitivity of $90 \%$, but this sensitivity is confined to the lesions of the lateral semicircular canal, and its accuracy has often been doubted. ${ }^{5)}$ The authors have experienced a case of labyrinthine fistula complicating chronic otitis

This is an Open Access article distributed under the terms of the Creative Commons Attribution Non-Commercial License (http://creativecommons. org/licenses/by-nc/3.0/) which permits unrestricted non-commercial use, distribution, and reproduction in any medium, provided the original work is properly cited. media with cholesteatoma, of which the initial nystagmus mimicked that of the lateral semicircular canal BPPV, and thus report it a possible explanation for the mechanism underlying the nystagmus pattern.

\section{Case Report}

A 61-year-old female patient was admitted to the emergency unit with the complaint of 2 days' dizziness of whirling nature accompanied by nausea and vomiting. The patient had medical history of hypertension and diabetes mellitus. Bedside neurologic examination revealed otherwise nonspecific. Brain diffusion weighted image (DWI) for the differential diagnosis of a central pathology was normal. On otoscopic examinations, normal right tympanic membrane was seen, but purulent ear discharge was filling the left external ear canal. The patient had been feeling a loss of hearing in her left ear for quite a long time and had been visiting a local otorhinolaryngologic clinic for several months diagnosed and treated for chronic external otitis in the left ear. The patient showed spontaneous, rightbeating horizontal nystagmus $(6.36 \mathrm{~d} / \mathrm{s})$. There was no gazeevoked nystagmus, and head thrust test was normal. Left-beating horizontal nystagmus $(9.70 \mathrm{~d} / \mathrm{s})$ was noted after head shaking. Dix-Hallpike test to either side provoked geotropic horizontal 
nystagmus, but the nystagmus was stronger on the left side ( $21.23 \mathrm{~d} / \mathrm{s}$ on the left side compared to $15.00 \mathrm{~d} / \mathrm{s}$ on the right) and so was the degree of symptoms. Geotropic direction-changing horizontal nystagmus was demonstrated on both sides during the supine roll test. The intensity of the nystagmus on both sides was comparable showing $17.71 \mathrm{~d} / \mathrm{s}$ on the right side and $17.03 \mathrm{~d} / \mathrm{s}$ on the left side. The latency and duration of positional nystagmus were 2 and 29 seconds, respectively on the right side, and 3 and 30 seconds, respectively on the left side. On the head bending nystagmus or lying-down nystagmus test, left-beating horizontal nystagmus $(7.00 \mathrm{~d} / \mathrm{s})$ on the head bending nystagmus test and right-beating horizontal nystagmus $(14.93 \mathrm{~d} / \mathrm{s})$ on the lying-down nystagmus test was demonstrated, respectively (Fig. 1). The bithermal caloric test response demonstrated unilateral canal paresis of $29.98 \%$ on the left side. These findings collectively led to the initial impression of geotropic variant of the left lateral semicircular canal BPPV on the left side combined with ipsilesional external otitis. The patient received the modified Lempert maneuver for treatment of the presumptive BPPV and was prescribed with empirical topical and oral antibiotics for management of the external otitis. She was discharged from the emergency unit for an our clinic visit scheduled for the following day.

At the next follow-up visit, the patient still complained of a positional vertigo aggravated on a leftward head turn, despite the therapeutic maneuver. A neurootologic examination revealed weak spontaneous, right-beating horizontal nystagmus. After head shaking, the horizontal nystagmus was seen to beat to the left. The patient was negative for positional nystagmus from the Dix-Hallpike test and supine roll test, but left-beating horizontal nystagmus on the head bending nystagmus test and right-beating horizontal nystagmus on the lyingdown nystagmus test was demonstrated, respectively. From these atypical nystagmus patterns, a thorough examination of the ear was undertaken, where keratinous debris and inflammatory polyps were hidden behind the purulent material blocking the left external auditory canal. The keratinous debris was cleared and polypoid tissues were removed for tissue pathology, after which the patient was sent for the TBCT. On the TBCT, the left middle ear cavity was mostly occupied with soft tissue eroding all the ossicles as well as the bone overlying the lateral semicircular canal and the tegmen tympani (Fig. 2). Magnetic resonance imaging and brain DWI were taken but reported negative for intradural intracranial invasion.

Under the diagnosis of left cholesteatomatous chronic otitis media with the complication of labyrinthine fistula, the patient was admitted to the hospital. The patient was instructed an absolute bed rest in a 30 degree head elevated position. A brain-blood-barrier-penetrating intravenous antibiotic and osmotic laxative were prescribed. Pure tone audiometry, taken
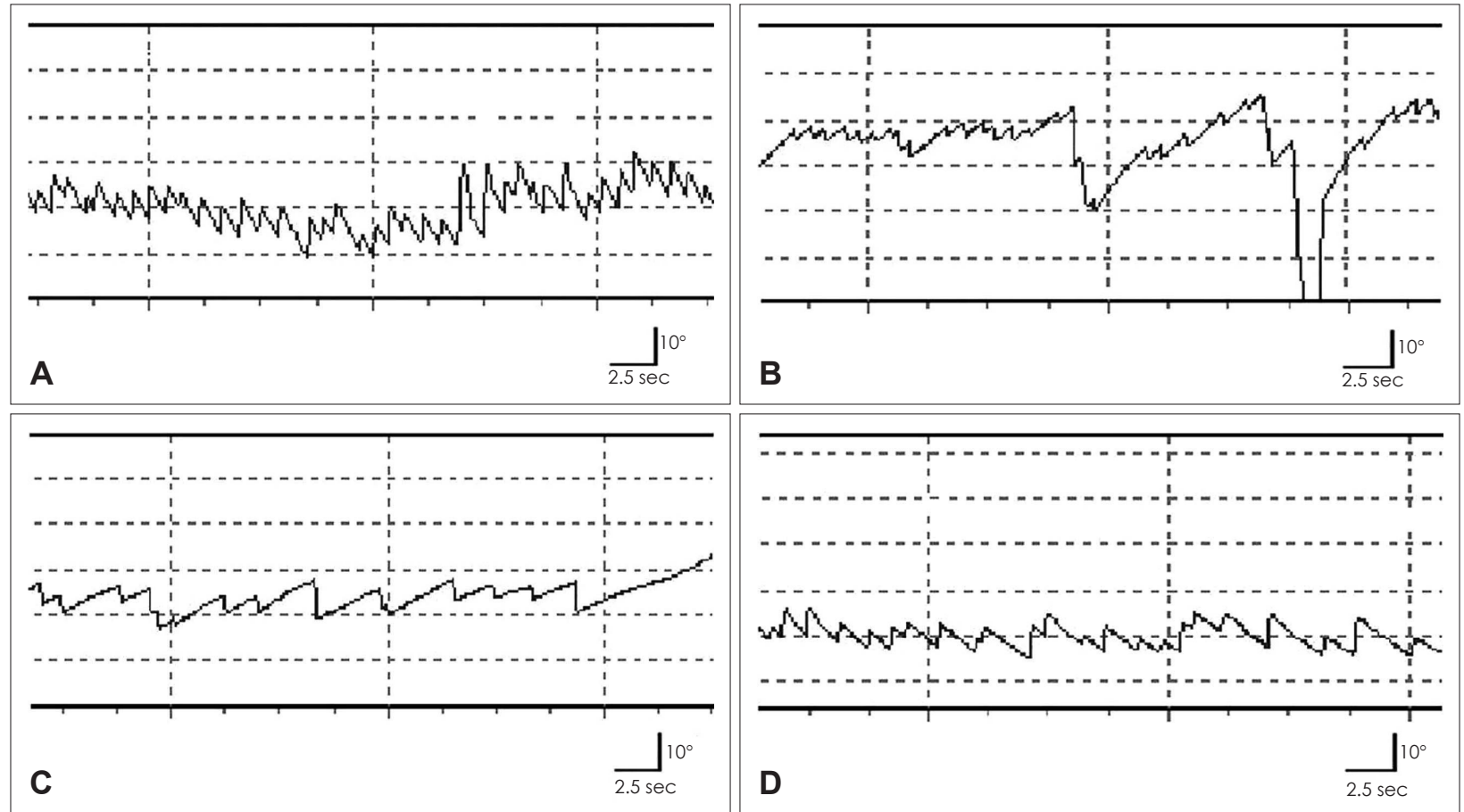

Fig. 1. Results of video-oculographic recordings. A: Geotropic positional nystagmus appears when the head is turned to the right in the supine position. B: Geotropic positional nystagmus appears when the head is turned to the left in the supine position. C: Left horizontal nystagmus appears when the head bent 30 degrees forward in the neutral sitting position. D: Right horizontal nystagmus appears when the patient lies supine with the head flexed 30 degrees forward. 
Fig. 2. Axial and coronal scans of temporal bone computed tomography (TBCT). Axial (A) and coronal (B) views of TBCT reveals a soft tissue density in the left middle ear cavity. Erosion of the lateral semicircular canal by assumed cholesteatoma can be seen in the left temporal bone (white arrows).
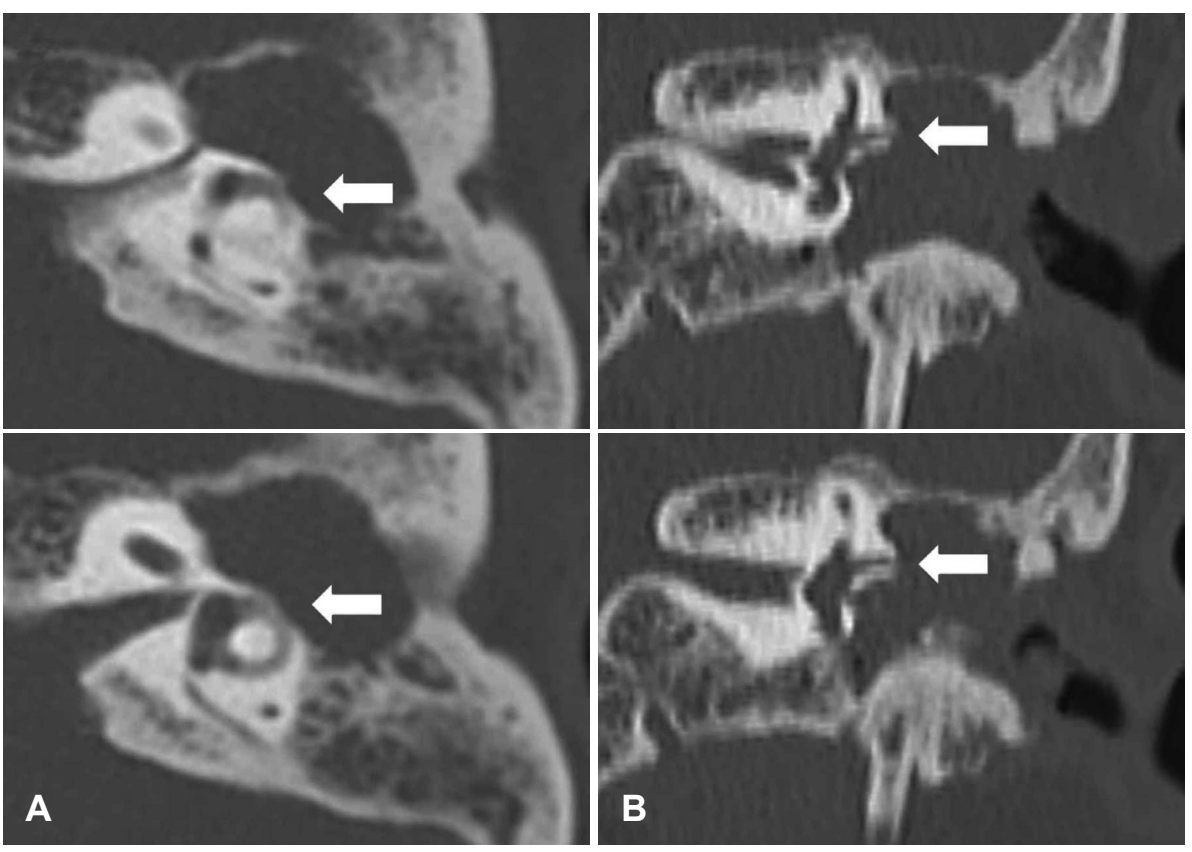

on the day of admission, showed normal air-conduction threshold of $18 \mathrm{~dB} \mathrm{HL}$ for the right ear, and a mixed hearing loss with an air-conduction threshold of $78 \mathrm{~dB}$ HL for the left ear (Fig. 3A). Tissue pathology taken on the first clinic exam was reported as a cholesteatoma. After resolution of dizziness, canal wall-down tympanomastoidectomy was performed for complete removal of the cholesteatoma on the 7th day of admission. Intraoperatively, dehiscence of the bony labyrinth of the lateral semicircular canal was observed without any leakage of perilymphatic fluid after the removal of the matrix. The dehiscence was covered by bone paste and muscle fascia. In the immediate postoperative period, the patient complained of dizziness, and a weak right-beating horizontal nystagmus was observed. Otherwise, it was uneventful postoperatively, free from signs of facial palsy or cerebrospinal fluid leaks. One day after the surgery, dizziness was subsided and there was no evidence of spontaneous nystagmus. The patient was discharged from the hospital on the 8th postoperative day without any evidence of complications. On follow up 3 months postoperatively, the patient was free of dizziness and felt improved hearing, of which pure tone audiogram showed improved hearing thresholds (left air-conduction threshold of $45 \mathrm{~dB}$ and boneconduction threshold of $23 \mathrm{~dB}$ ) (Fig. 3B).

\section{Discussion}

Labyrinthine fistula is an aberrant connection between the perilymph-filled inner ear and air-filled middle ear, of which the perilymph leak results in pathology of the vestibule and the cochlea. ${ }^{6}$ In most instances, bony labyrinth is involved up to but not involving the endosteum so that the endosteum is merely separating the perilymphatic space from the matrix. ${ }^{7)}$ In more rare cases, the granulation or fibrous tissue may infiltrate the membranous labyrinth, thus some authors have described a three-stage classification of labyrinthine fistulas. ${ }^{8}$

In this case, we initially suspected the patient to have a central pathology because of the inverted head shaking nystagmus beating to the opposite direction from the spontaneous nystagmus. However, no central lesions were observed on the brain DWI. On both the Dix-Hallpike test and supine roll test in the emergency unit, substantial geotropic direction-changing horizontal nystagmus was provoked. Head bending nystagmus was beating to the left, and lying-down nystagmus was to the right. These findings, collectively, added on the findings of purulent otorrhea with a history of recent treatment of external otitis, misled to an initial presumptive diagnosis of the left lateral semicircular canal BPPV and external otitis. Although the nystagmus was more intense on the right supine roll compared to the left, the difference was not significant, and the symptoms were similar between both sides. Moreover, the head bending nystagmus was seen to beat to the left, leading to the impression of the lateral semicircular canal BPPV on the left side rather than the right. ${ }^{9}$ However, in the follow up clinic visit the next day, the diagnosis was corrected to be a left labyrinthine fistula complicating a cholesteatoma on observing an atypical nystagmus pattern and clearing out the external ear of purulent and keratinous materials.

The nature of the nystagmus pattern in this patient with labyrinthine fistula mimicking the typical positioning nystagmus of the left semicircular canal BPPV requires some explana- 

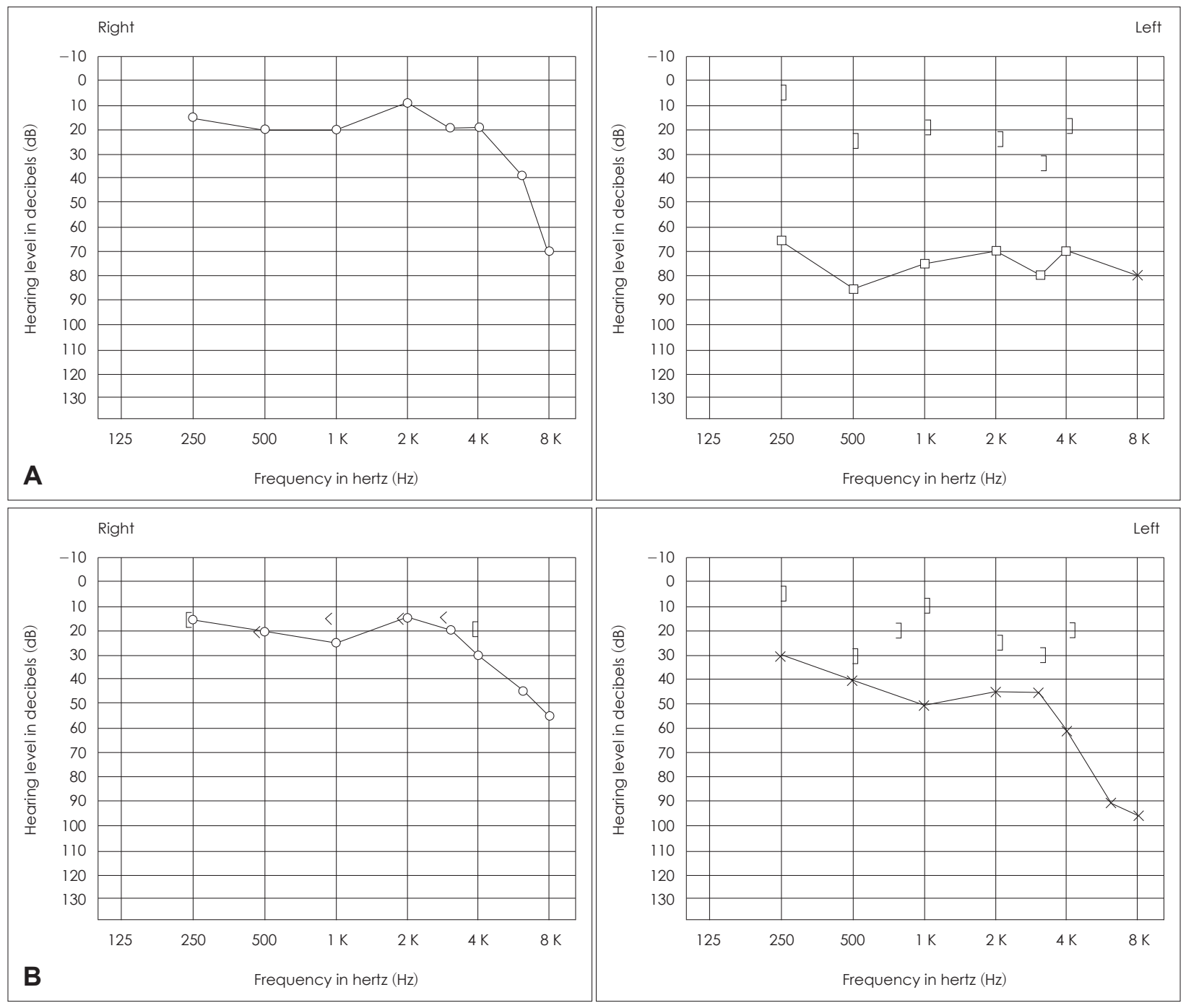

Fig. 3. Pure tone audiometry. A: On the first day of outpatient visit, pure tone audiometry shows conductive hearing loss in the left ear. B: Three months after operation, pure tone audiometry shows a substantial improvement in the left ear.
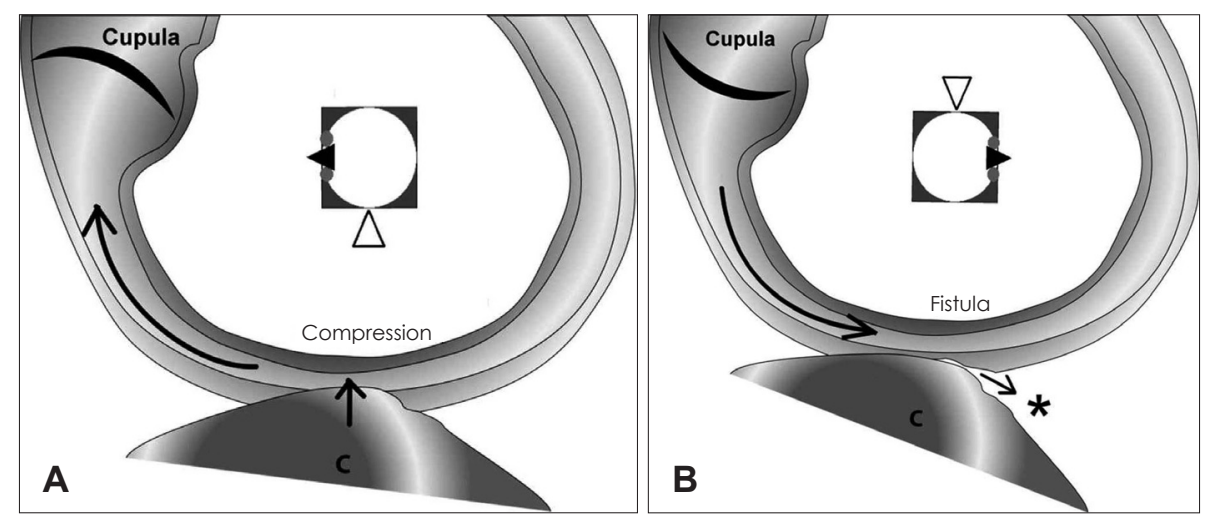

Fig. 4. Hypothesis of directional changing positional nystagmus. Cholesteatoma causes an erosive loss of the endochondral bone overlying the labyrinth. A: When the head turns to the left side in the supine position, cholesteatoma may become more attached to left horizontal semicircular canal. It may evoke mass-induced positive pressure (straight arrow) of the membranous labyrinth, which causes utriculopetal flow of endolymphatic fluid. It may cause utriculopetal deviation of the cupula leading to an excitatory stimuli. B: When the head turns to the right side in the supine position, cholesteatoma may be detached to left horizontal semicircular canal. It can evoke a slight leakage of perilymphatic fluid based on the Pascal's principle, which causes utriculofugal flow of endolymphatic fluid. It may causes utriculofugal deviation of the cupula leading to an inhibitory stimuli. *slit leakage of perilymphatic fluid. Curved arrow: direction of endolymphatic flow, white arrowhead: lesion side, black arrowhead: patient's nose. C: cholesteatoma. 
tion. Subtle movements of the cholesteatoma following positional changes of the head may affect pressure changes in the area of the fistula, which in turn influence the change in the direction of the endolymphatic flow. When the head was turned 90 degrees to the left during the initial examination in the emergency unit, the cholesteatoma might have leaned closer to the left semicircular canal creating a positive pressure on the membranous labyrinth. Hence, a utriculopetal endolymphatic flow causing a utriculopetal deviation of the cupula in turn, leads to an excitatory stimuli creating a left-beating geotropic horizontal nystagmus in accordance with Ewald's second law (Fig. 4A). ${ }^{10)}$ When there is a rightward 90 degrees head rotation, the cholesteatoma moves away from the lateral semicircular canal creating a space between the bony labyrinth and the cholesteatoma, within which a negative pressure is formed. This negative pressure creates utriculofugal endolymphatic flow and a resultant rightward geotropic horizontal nystagmus from utriculofugal cupula deviation and an inhibitory stimulus (Fig. 4B). Pascal's Law may explain for the pressure changes and the directions of the endolymphatic flow. ${ }^{11)}$

Most acutely vertiginous patients are easily diagnosed with otologic pathologies such as BPPV, Meniere's disease or acute vestibular neuritis based upon patient's symptoms and simple audio-vestibular workups. But we experienced a misdiagnosed dizzy patient by paying less attention to physical findings and being presumptive on initial video-oculographic results. Since the fistula test is of little value in the diagnosis of labyrinthine fistula, negative test results might have added to the missed initial diagnosis. ${ }^{12,13)}$ Moreover, fistula test may not be applied in cases where a perforated tympanic membrane with acute inflammation might be expected. Our case is an example where clinical diagnosis of a dizzy patient may be misleading if made based only on presumptive judgments without a thorough otologic workup and thereby report the case with a possible explanation for the complexity and equivocality of the nystagmus study results throughout the course of diagnosis.

\section{REFERENCES}

1) Turner B, Eynon-Lewis N. Systematic approach needed to establish cause of vertigo. Practitioner 2010;254:19-23, 2-3.

2) Palva T, Kärjä J, Palva A. Opening of the labyrinth during chronic ear surgery. Arch Otolaryngol 1971;93:75-8.

3) Kim CS, Choi BY, Hwang CH, Ahn SH, Park JB, Koo JW, et al. Clinical presentation and management of labyrinthine fistula in chronic otitis media with cholesteatoma. Korean J Otolaryngol-Head Neck Surg 2002;45:1039-45.

4) Maitland CG. Perilymphatic fistula. Curr Neurol Neurosci Rep 2001; 1:486-91.

5) Jung HW, Park MH, Kang JG, Song BH, Koo JW, Chang SO, et al. Diagnostic efficacy of high resolution computerized tomography (HRCT) on labyrinthine fistula. Korean J Otolaryngol-Head Neck Surg 2001;44:37-40.

6) Pfaltz CR. Complications of otitis media. ORL J Otorhinolaryngol Relat Spec 1982;44:301-9.

7) Gacek RR. The surgical management of labyrinthine fistulae in chronic otitis media with cholesteatoma. Ann Otol Rhinol Laryngol 1974; 83:Suppl 10:1-19.

8) Dornhoffer JL, Milewski C. Management of the open labyrinth. Otolaryngol Head Neck Surg 1995;112:410-4.

9) Lee SH, Choi KD, Jeong SH, Oh YM, Koo JW, Kim JS. Nystagmus during neck flexion in the pitch plane in benign paroxysmal positional vertigo involving the horizontal canal. J Neurol Sci 2007;256:75-80.

10) Brandt T. Benign paroxysmal positional vertigo. In: Brandt T, editor. Vertigo: its multisensory syndrome. 2nd ed. London: Verlag-Springer;1999. p.269-79.

11) Srivastava A, Sood A, Joy SP, Woodcock J. Principles of physics in surgery: the laws of flow dynamics physics for surgeons - Part 1. Indian J Surg 2009;71:182-7.

12) Busaba NY. Clinical presentation and management of labyrinthine fistula caused by chronic otitis media. Ann Otol Rhinol Laryngol 1999;108:435-9.

13) Soda-Merhy A, Betancourt-Suárez MA. Surgical treatment of labyrinthine fistula caused by cholesteatoma. Otolaryngol Head Neck Surg 2000;122:739-42. 